\title{
Upregulation of miR-129-5p increases the sensitivity to Taxol through inhibiting HMGB1-mediated cell autophagy in breast cancer MCF-7 cells
}

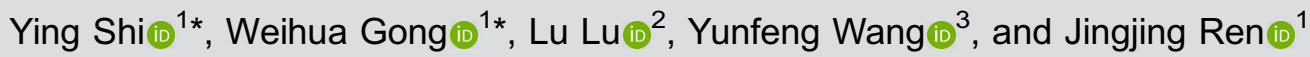 \\ ${ }^{1}$ Clinical Laboratory, The Third Affiliated Hospital of Zhengzhou University, Zhengzhou, China \\ ${ }^{2}$ Clinical Laboratory, Beijing Chaoyang Emergency Medical Center, Beijing, China \\ ${ }^{3}$ Clinical Laboratory, Xiang Cheng City First Person Hospital, Zhoukou, China
}

\begin{abstract}
Although Taxol has improved the survival of cancer patients as a first-line chemotherapeutic agent, an increasing number of patients develop resistance to Taxol after prolonged treatment. The potential mechanisms of cancer cell resistance to Taxol are not completely clear. It has been reported that microRNAs (miRNAs) are involved in regulating the sensitivity of cancer cells to various chemotherapeutic agents. In this study, we aimed to explore the role of miR-129-5p in regulating the sensitivity of breast cancer cells to Taxol. Cell apoptosis and autophagy, and the sensitivity of MCF-7 cells to Taxol were assessed with a series of in vitro assays. Our results showed that the inhibition of autophagy increased the Taxol-induced apoptosis and the sensitivity of MCF-7 cells to Taxol. Up-regulation of miR-129-5p also inhibited autophagy and induced apoptosis. Furthermore, miR-129-5p overexpression increased the sensitivity of MCF-7 cells to Taxol. High mobility group box 1 (HMGB1), a target gene of miR-129-5p and a regulator of autophagy, was negatively regulated by miR-129-5p. We found that interference of HMGB1 enhanced the chemosensitivity of Taxol by inhibiting autophagy and inducing apoptosis in MCF-7 cells. Taken together, our findings suggested that miR-129-5p increased the chemosensitivity of MCF-7 cells to Taxol through suppressing autophagy and enhancing apoptosis by inhibiting HMGB1. Using miR-129-5p/HMGB1/autophagy-based therapeutic strategies may be a potential treatment for overcoming Taxol resistance in breast cancer.
\end{abstract}

Key words: miR-129-5p; HMGB1; Autophagy; Taxol; Breast cancer

\section{Introduction}

Breast cancer is the most common cancer-related cause of death in females worldwide (1). Although surgery and radiotherapy can be used for the treatment of breast cancer, chemotherapy is still common and essential and can reduce clinical stage, kill or reduce micrometastases, and prevent distant metastases (2,3). Taxol is among the most widely used chemotherapy agents in the treatment of breast cancer (4). It stabilizes microtubules, leading to cell cycle arrest and, ultimately, cell death (5-7). Nevertheless, intrinsic and acquired drug resistance to Taxol remains an important factor limiting the clinical efficacy $(8,9)$. Thus, it is important to enhance the sensitivity of breast cancer to Taxol. However, the potential mechanisms for the development of Taxol resistance are still unclear.

Micro RNAs (miRNAs) are a class of short, endogenous, non-coding RNAs ( $\sim 20-24$ nucleotides) that regulate the expression of a diversity of genes (10). They have been identified to be involved in a variety of biological processes (11-13). Recently, an increasing number of studies have reported that dysregulation of miRNAs might be related to drug resistance (14-16). With the deepening of miRNA research, targeting their downstream genes to regulate multidrug resistance has become a research hotspot. MiR-129-5p, a tumor inhibitor, has been validated to inhibit cancer cells migration, proliferation, differentiation, and epithelial-mesenchymal transition (17-20). In addition, miR-129-5p-mediated beclin-1 suppression inhibits endothelial cell autophagy in atherosclerosis (21). With the in-depth study on the relationship between miRNAs and cancer resistance (22), more and more researchers consider that miR-129-5p is an important molecule involved in cancer resistance (23). Regulating the expression of miR-129-5p might be a potential strategy for the treatment of cancer resistance.

Correspondence: Ying Shi: <15037169625@126.com>

${ }^{*}$ These authors contributed equally to this work.

Received April 10, 2019 | Accepted September 11, 2019 
Autophagy is a well-known important protective mechanism that can help cells against adverse growth and promote cell survival $(24,25)$. Protective autophagy can resist chemotherapeutic-induced cell apoptosis and cell injury in the course of anti-tumor treatment $(26,27)$. High mobility group box 1 (HMGB1) is a regulator of autophagy as a ubiquitous nuclear protein, which can regulate and promote various DNA-related activities such as transcription, replication, and repair (28-30). Recently, studies indicated that HMGB1 plays an essential role in chemotherapy sensitivity through modulating protective autophagy in a number of cancers, including breast cancer $(31,32)$. Thus, targeting HMGB1-mediated autophagy might improve the chemosensitivity of cancer cells. Luo et al. (33) reported that miR-129-5p regulated autophagy by targeting HMGB1 in breast cancer MCF-7 cells. However, no study has focused on the association of miR-129-5p dysregulation and the sensitivity of breast cancer cells to Taxol by targeting HMGB1.

\section{Material and Methods}

\section{Cell culture and drug treatment}

MCF-7 cells were purchased from the American Type Culture Collection (ATCC, USA). MCF-7 cells were maintained in RPMI-1640 (Solarbio, China) medium containing $10 \%$ fetal bovine serum at $37^{\circ} \mathrm{C}$ and $5 \% \mathrm{CO}_{2}$. MCF-7 cells were cultured two days before treatments. Cells were treated with 3-methyladenine (3-MA; Selleck, USA) an autophagy inhibitor, for $2 \mathrm{~h}$ before Taxol (Xi'an Haoxuan Bio-tech Co., Ltd., China) treatment for $24 \mathrm{~h}$.

\section{Cell proliferation assay}

MCF-7 cells were seeded onto 96-well cell culture cluster plates at a density of $8 \times 10^{3}$ cells/well in $100 \mu \mathrm{L}$ RPMI 1640 medium (Solarbio, China) supplemented with $10 \%$ fetal bovine serum (Gibco BRL, USA) and grown overnight. After treatment with different concentrations $(3.9,7.8,15.6,31.2,125.0,500.0$, and $1000.0 \mathrm{nM})$ of Taxol for $24 \mathrm{~h}$, cell proliferation was measured using the Cell Counting Kit-8 (Dojindo, China) according to the manufacturer's protocol. The results are reported as a ratio of cell proliferation/inhibition and the experiments were repeated at least three times. Inhibition of proliferation rate $(\%)=(1-A b$ (absorbance) value of experimental group / $\mathrm{Ab}$ value of control group) $\times 100 \%$.

\section{miRNA and siRNA transfection}

MiR-129-5p mimics (miR-129-5p), miRNA control (miR-NC), small-interfering RNA HMGB1 (si-HMGB1), and small-interfering RNA control (si-NC) were purchased from Sangon Biotech (China). They were transfected with LipoHigh reagent (Sangon Biotech) into MCF-7 cells according to the manufacturer's instructions. Cell lysates were prepared for subsequent experiments after transfection.

Total RNA isolation from MCF-7 cells was performed using Trizol (Sangon Biotech). cDNA was synthesized using Revert Acc kit (Sangon Biotech) according to the manufacturer's instructions. Quantitative real-time PCR was performed on an $\mathrm{ABI} 7500$ real-time system (Applied Biosystems, USA) according to the manufacturer's protocol. Data were calculated using the $2^{-\Delta \Delta C T}$ method based on the internal control. The relative expression of miR129-5p was normalized to U6 expression. The relative expression of HMGB1 mRNA was normalized to GAPDH expression. The data set was generated by at least three independent experiments. PCR primers are shown in Table 1.

\section{Western blotting analysis}

MCF-7 cells were lysed with RIPA lysis buffer (Solarbio), and western blotting was performed using standard procedures. The concentration of protein was determined using the BCA protein quantitation kit (Sangon Biotech). Equal quantities of denatured protein samples were resolved on $12 \%$ SDS-polyacrylamide gels based on the targeted molecular weight and then transferred onto PVDF membranes (Millipore, USA). Membranes were incubated with a specific primary antibody against HMGB1 (1:10000 dilution; Abcam, USA), p62 (1:10000 dilution; Abcam), and LC3B (1:1000 dilution; Cell Signaling Technology, USA). Proteins were visualized using ECL system (Millipore). GAPDH was used as an internal control.

\section{Flow cytometry for apoptosis}

MCF-7 cells were seeded overnight in six-well plates. Cells were transfected with siRNA-HMGB1, miR-129-5p, or control for $24 \mathrm{~h}$ and then treated with $\mathrm{IC}_{30}$ Taxol. After $24 \mathrm{~h}$, cells were harvested and stained with annexin V-FITC and propidium iodide (PI) following the manufacturer's protocol (BD Biosciences, USA). Finally, stained

Table 1. Polymerase chain reaction primers.

\begin{tabular}{|c|c|c|}
\hline Name & Forward primer & Reverse primer \\
\hline miR-129-5p & 5'-CTTTTTGCGGTCTGGGCTTG-3' & 5'-AACGCTTCACGAATTTGCGT-3' \\
\hline U6 & 5'-CTCGCTTCGGCAGCACA-3' & 5'-AACGCTTCACGAATTTGCGT-3' \\
\hline HMGB1 & 5'-ACAAGGCCCGTTATGAAAGA-3' & 5'-GAAGAGGAAGAAGGCCGAAG-3' \\
\hline GAPDH & 5'-CAGGAGGCATTGCTGATGAT-3' & 5'-GAAGGCTGGGGCTCATTT-3' \\
\hline
\end{tabular}


cells were analyzed by flow cytometry (BD FACSCanto, BD Biosciences). Three biological experiments were performed.

\section{Immunofluorescence}

First, MCF-7 cells were seeded onto cover-slips and then fixed in $4 \%$ paraformaldehyde for $30 \mathrm{~min}$ at room temperature. Cells were washed with PBS three times, blocked with 5\% goat serum (Ding Guo Chang Sheng, China), and permeabilized with $0.3 \%$ Triton X-100 for $15 \mathrm{~min}$. Cells were incubated with rabbit polyclonal antiHMGB1 (1:200 dilution, Abcam) at $4^{\circ} \mathrm{C}$ overnight. After washing in PBS, cells were incubated with FITC-labeled anti-rabbit secondary antibody (Ding Guo Chang Sheng) for $1 \mathrm{~h}$ at room temperature. Images were acquired using a fluorescence microscope (Olympus, Japan).

\section{Bioinformatics analysis}

Overlapping target genes were predicted using miRDB (mirdb.org), microRNA.org, and TargetScan (targetscan. org). The punitive binding sites among miR-129-5p and HMGB1 were predicted using TargetScan. The expression of HMGB1 in normal breast tissue and breast cancer tissue were searched in the online databases Oncomine (https://www.oncomine.org/resource/login.html) and the Human Protein Atlas (https://www.proteinatlas.org/).

\section{Statistical analysis}

Data were analyzed using SPSS 21.0 statistical software (IBM, USA). All results are reported as means \pm SD. Student's $t$-test or one-way ANOVA were used to analyze the differences between treatment groups. $\mathrm{P}<0.05$ was considered statistically significant.

\section{Results}

The sensitivity of MCF-7 cells to Taxol was increased by inhibiting autophagy and inducing apoptosis

First, we used the CCK- 8 assay to detect the inhibition of Taxol on MCF-7 cells proliferation. The results showed that $\mathrm{IC}_{25}$ of Taxol was approximately $31.2 \mathrm{nM}$ at $24 \mathrm{~h}$ (Figure 1A). Then, we cultured MCF-7 cells for 24,48 , and $72 \mathrm{~h}$ in $31.2 \mathrm{nM}$ Taxol. We found that the inhibition of Taxol on breast cancer MCF-7 cells depended on the time of Taxol treatment (Figure 1B). Last, we explored the relationship between autophagy and Taxol. LC3B was used as an important marker for autophagy. LC3B has two isoforms, LC3B-I and LC3B-II. When autophagy was up-regulated, LC3B-I was transformed into LC3B-II, and the ratio of LC3B-II/LC3B-I was increased. Therefore, the ratio of LC3B-II/LC3B-I can indicate the level of autophagy (34). As shown in (Figure 1C), the ratio of LC3B-II/LC3B-I decreased over time in $24 \mathrm{~h}$ after Taxol treatment. In addition, p62 can be degraded during the process of autophagy lysosome formation, thus the decrease in p62 usually signifies the occurrence of autophagy (35).
Figure 1D showed that the expression of $\mathrm{p} 62$ was increased in Taxol-treated MCF-7 cells. These results showed Taxol exerted its cytotoxicity by inhibiting autophagy, and the inhibition of autophagy might increase the sensitivity of MCF-7 cells to Taxol. Thus, we used 3-MA, a selective PI3K inhibitor, to verify the above results. As shown in Figure 2A, the ratio of LC3B-II/LC3B-I was lowest and the expression of p62 was highest in the Taxol combined with 3-MA group. It indicated that 3-MA improved the inhibition of autophagy by Taxol. Furthermore, our data showed that the rate of apoptosis (Figure 2B) and the inhibition of cell proliferation (Figure $2 \mathrm{C}$ ) by Taxol was increased in the Taxol-3-MA combined group. This indicated that the sensitivity of MCF-7 cells to Taxol and Taxolinduced cell apoptosis could be increased by inhibiting autophagy. Furthermore, these results suggested that the chemosensitivity of MCF-7 cells to Taxol can be improved by inhibiting autophagy and inducing apoptosis.

miR-129-5p enhanced chemosensitivity of Taxol by inhibiting autophagy and promoting apoptosis in MCF-7 cells

To explore whether miR-129-5p was involved in regulating the therapeutic effect of Taxol through the regulation of autophagy and apoptosis, we transfected miR-129-5p mimics into MCF-7 cells and then treated them with $31.2 \mathrm{~nm}$ of Taxol for $24 \mathrm{~h}$. As shown in Figure 3A, miR-129-5p overexpression significantly increased the relative expression of miR-129-5p in MCF-7cells. Compared with miRNA-NC transfected cells, we found that miR-129-5p overexpression suppressed the conversion of LC3B-I to LC3B-II and inhibited the degradation of p62 with or without Taxol treatment (Figure 3B). This data strongly suggested that miR-129-5p could increase the inhibition of Taxol to autophagy. Then, we investigated whether miR-129-5p overexpression could enhance Taxolinduced apoptosis using flow cytometry. As shown in Figure 3C, miR-129-5p overexpression increased Taxolinduced apoptosis. Finally, we examined the effect of miR-129-5p in Taxol chemosensitivity using CCK-8 assays. Results showed that coupled with different concentrations of Taxol for $24 \mathrm{~h}$, miR-129-5p overexpression significantly increased the inhibition of cell proliferation compared to the miR-NC group (Figure 3D). Taken together, these results support that miR-129-5p overexpression could increase the chemosensitivity of MCF-7 cells to Taxol by inhibiting autophagy and inducing apoptosis.

\section{HMGB1 was downregulated by miR-129-5p}

To decipher the potential mechanisms promoting chemosensitivity in human MCF-7 cells by miR-129-5p, we used TargetScan, miRDB, and microRNA online analysis tools to search for the potential target genes of miR-129-5p. We found that there were eight overlapping target genes of miR-129-5p (Supplementary Figure S1A). Since HMGB1 is a unique regulator for autophagy among 
A

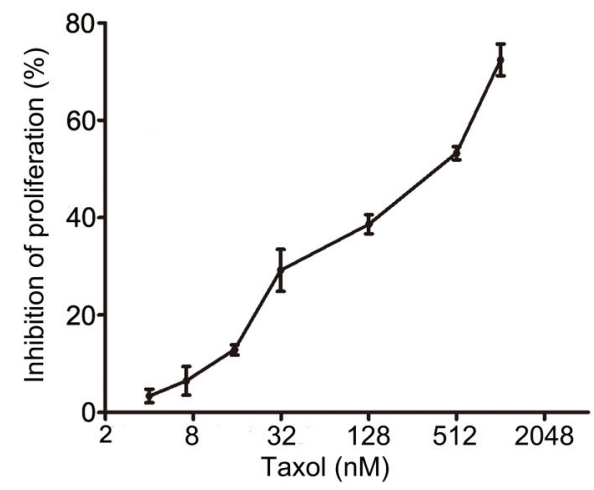

C

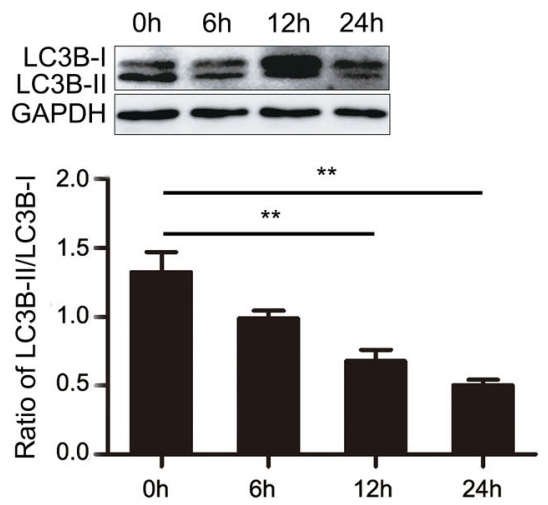

B

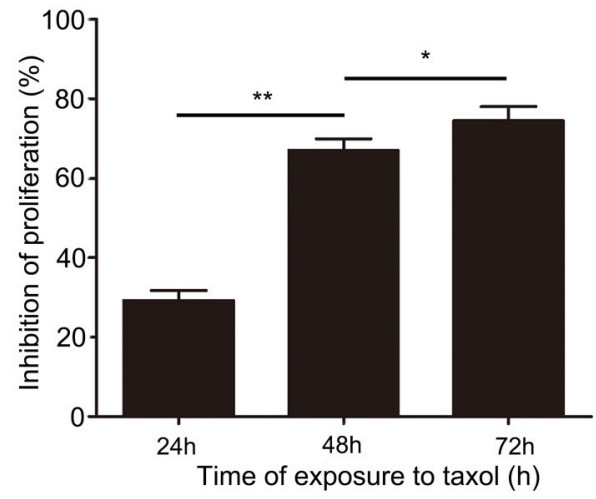

D

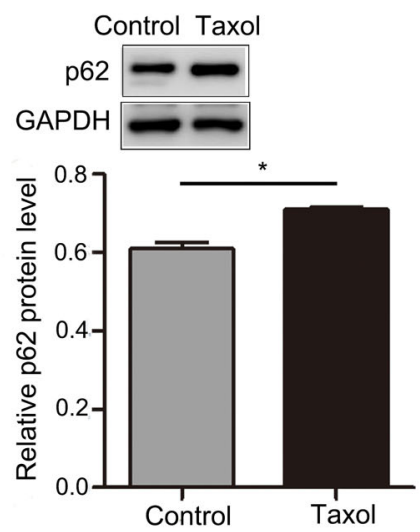

Figure 1. A, Cell proliferation was determined by the CCK-8 assay after treatment with different concentrations of Taxol for $24 \mathrm{~h}$. B. Cell proliferation was determined by the CCK-8 assay after treatment with $31.2 \mathrm{nM}$ Taxol at different time-points. C, Expression of LC3B-I and LC3B-II in MCF-7 cells was determined by western blotting after treatment with $31.2 \mathrm{nM}$ Taxol at different time-points. D, p62 expression in MCF-7 cells was determined by western blotting after treatment with $31.2 \mathrm{nM}$ Taxol for $24 \mathrm{~h}$. Data are reported as means \pm SD of three independent experiments. ${ }^{*} \mathrm{P}<0.05$, ${ }^{* *} \mathrm{P}<0.01$, vs control group (ANOVA).

these eight overlapping target genes, we focused on researching HMGB1. The online database TargetScan indicated that there were two possible binding sites among miR-129-5p and HMGB1 (Supplementary Figure S1B). We also found that the expression of HMGB1 was higher in breast cancer tissue compared to normal breast tissue using the tumor database of Oncomine (Supplementary Figure S1C) and the Human Protein Atlas (Supplementary Figure S1D).

To validate the effect of miR-129-5p on endogenous expression of HMGB1, we determined the levels of HMGB1 by qRT-PCR and western blotting in miR-129-5p transfected cells. Our results showed that miR-129-5p overexpression inhibited the expression of HMGB1 both at the mRNA (Figure 4A) and protein (Figure 4B) levels in MCF-7 cells. We also analyzed the inhibition effect of miR-129-5p on HMGB1 protein by immunofluorescence assay (Figure 4C). The results were consistent with Figure 4B, where the fluorescence intensity of HMGB1 was lowest in miR-129-5p transfected cells. These data indicated that miR-129-5p directly inhibited the endogenous expression of HMGB1 in MCF-7 cells.

\section{Downregulation of HMGB1 enhanced the chemosensitivity of Taxol by inhibiting autophagy and promoting apoptosis in MCF-7 cells}

Based on the association of miR-129-5p and HMGB1, we hypothesized that miR-129-5p may inhibit autophagy and promote apoptosis through regulating HMGB1. We transfected si-HMGB1 or si-NC into MCF-7 cells and then treated with $31.2 \mathrm{nM}$ Taxol to determine the function of HMGB1. First, our results showed that si-HMGB1 inhibited the expression of HMGB1 on both the mRNA (Figure 5A) and protein levels (Figure $5 B$ ). Subsequently, we detected the level of autophagy by western blotting. As shown in Figure $5 \mathrm{C}$, the ratio of LC3B-II/LC3B-I was lowest in the transfected si-HMGB1 Taxol combined treatment group, but the expression of p62 was highest. These data indicated that downregulation of HMGB1 enhanced the inhibition of Taxol to autophagy. Furthermore, the results 
A
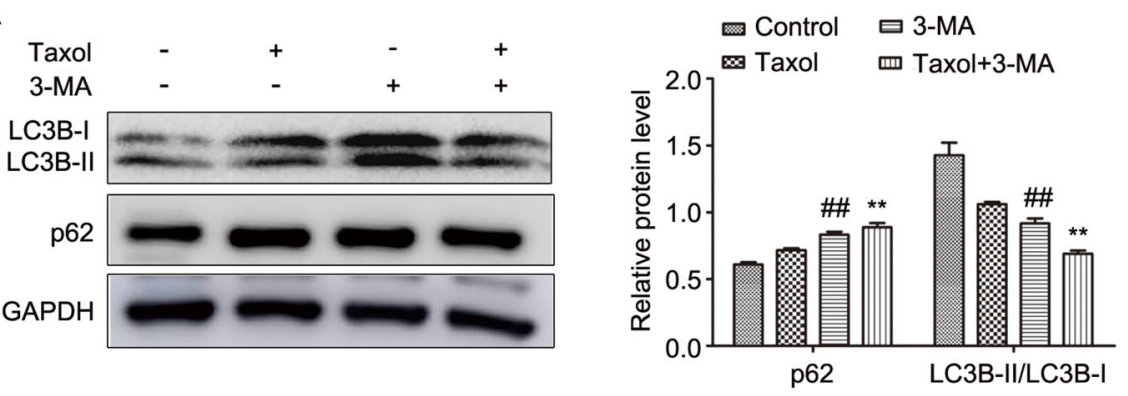

B

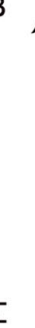
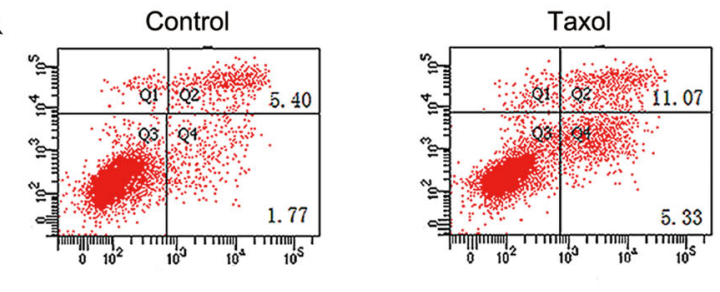

$\bar{\alpha}$
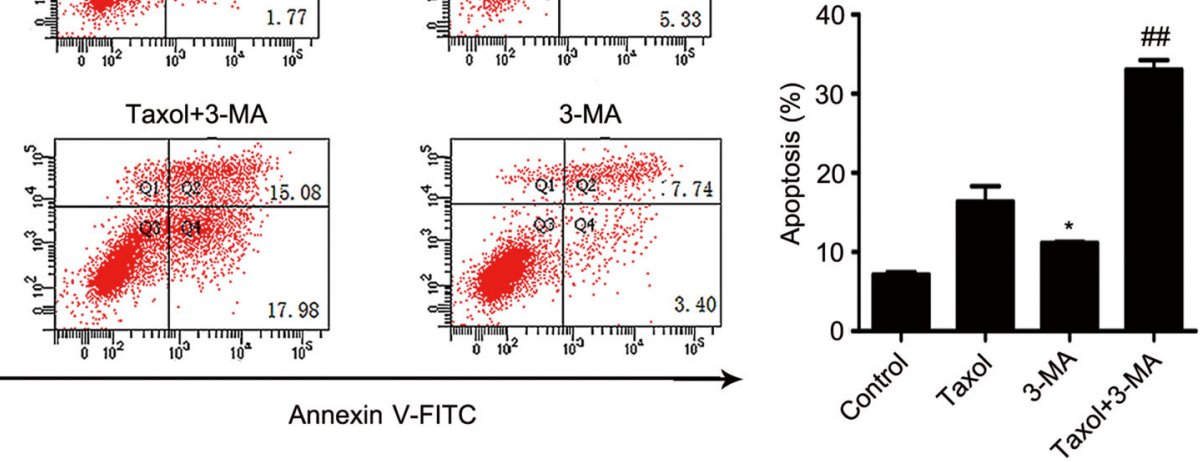

C

Annexin V-FITC

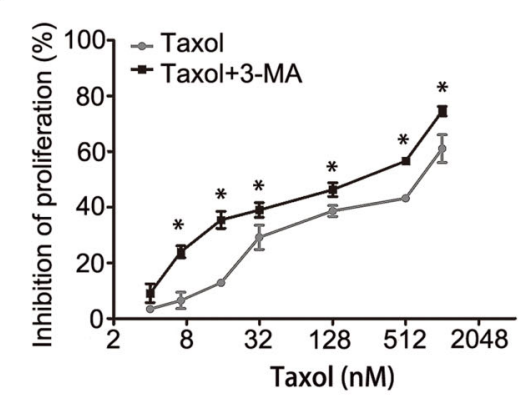

Figure 2. A and B, MCF-7 cells were treated with $5 \mathrm{mM}$ 3-MA for 2 hours before $31.2 \mathrm{nM}$ Taxol treatment for $24 \mathrm{~h}$. LC3B-I, LC3B-II, and p62 expression in cells was determined by western blotting and cellular apoptosis was determined by flow cytometry. C, Cell proliferation was determined by the CCK-8 assay after pre-treatment with $5 \mathrm{mM} 3-\mathrm{MA}$ for $2 \mathrm{~h}$ and different concentrations of Taxol for $24 \mathrm{~h}$. Data are reported as means $\pm \mathrm{SD}$ of three independent experiments. ${ }^{*} \mathrm{P}<0.05,{ }^{* *} \mathrm{P}<0.01$, vs control group; ${ }^{\# \#} \mathrm{P}<0.01$, vs Taxol group (ANOVA).

of flow cytometry demonstrated that downregulation of HMGB1 could increase Taxol-induced apoptosis (Figure 6A). Finally, the inhibition of cell proliferation was determined by CCK-8 after transfection with si-HMGB1 and exposure to different concentrations of Taxol for $24 \mathrm{~h}$. The data demonstrated that si-HMGB1 increased the rate of Taxolinhibited cell proliferation (Figure 6B). This suggested that the downregulation of HMGB1 could enhance the chemosensitivity of MCF-7 cells to Taxol by inhibiting autophagy and promoting apoptosis.

\section{Discussion}

As an important chemotherapeutic agent for the treatment of cancer, including breast cancer, Taxol has successfully improved the survival of patients (7). However, some patients still have lower Taxol sensitivity because of drug resistance, which leads to poor prognosis of patients (8). Recently, altered levels of autophagy and miRNAs were attractive candidates for study as regulators of chemosensitivity in breast cancer $(11,16,26)$. In the 
A

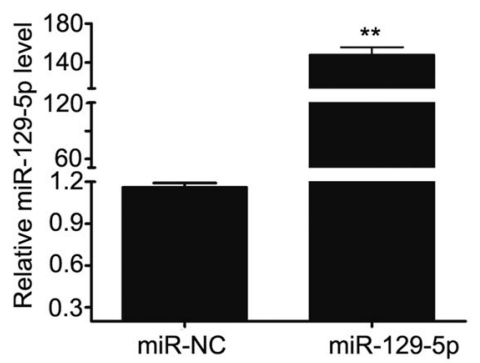

B
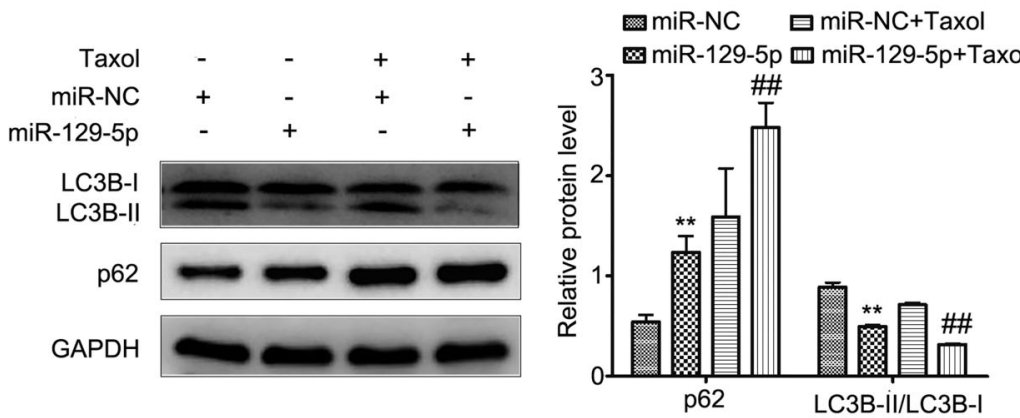

C
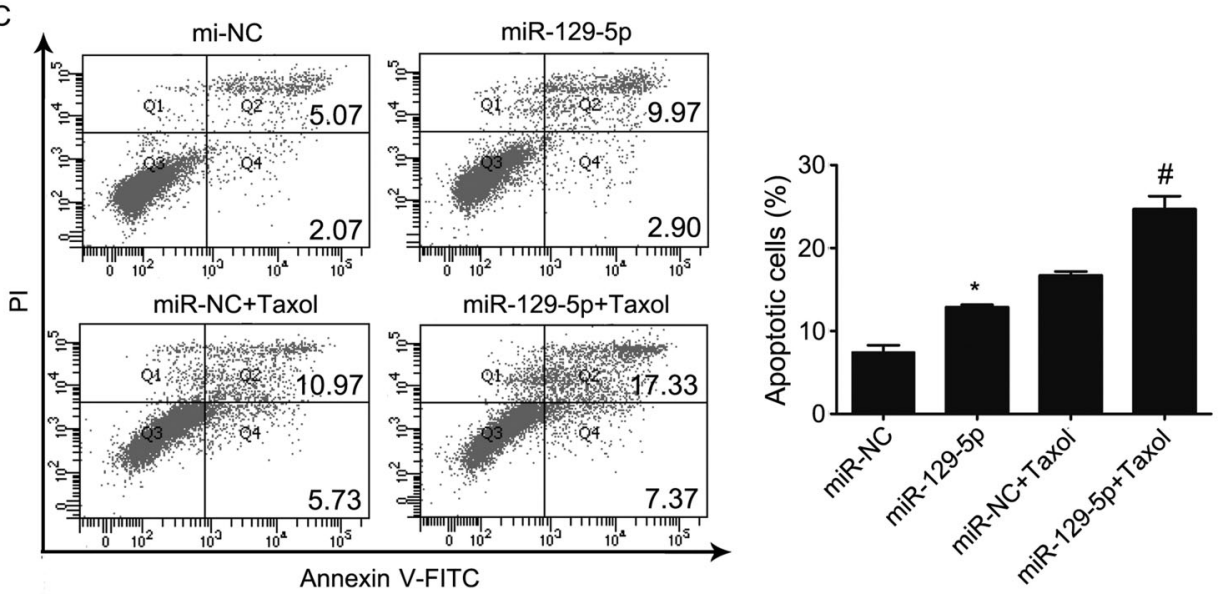

D

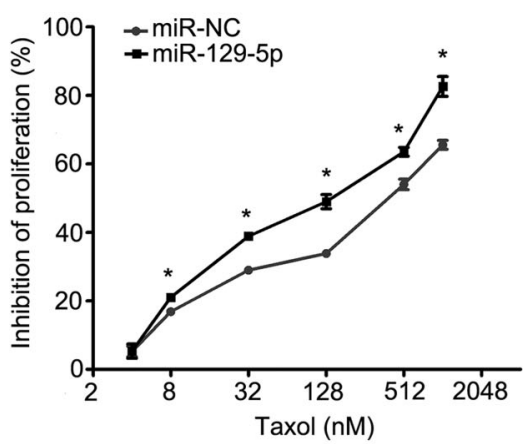

Figure 3. A, Relative miR-129-5p expression was detected by qRT-PCR analysis in MCF-7 cells transfected with miR-129-5p mimics or miR-NC. MiR-NC acted as a negative control. B and C, Cells were transfected with miR-NC or miR-129-5p mimics and then treated with $31.2 \mathrm{nM}$ Taxol for $24 \mathrm{~h}$. B, LC3B-I, LC3B-II, and p62 expression in MCF-7 cells were determined by western blot. C, Cellular apoptosis was determined by flow cytometry. D, Cell proliferation was determined by the CCK-8 assay after transfection with miR-NC or miR-129-5p mimics and treatment with different concentrations of Taxol for $24 \mathrm{~h}$. Data are reported as the means \pm SD of three independent experiments. ${ }^{*} \mathrm{P}<0.05$, ${ }^{*} \mathrm{P}<0.01$, vs miR-NC group; ${ }^{\#} \mathrm{P}<0.05$, ${ }^{\# \#} \mathrm{P}<0.01$, vs miR-NC + Taxol group (ANOVA). 
A
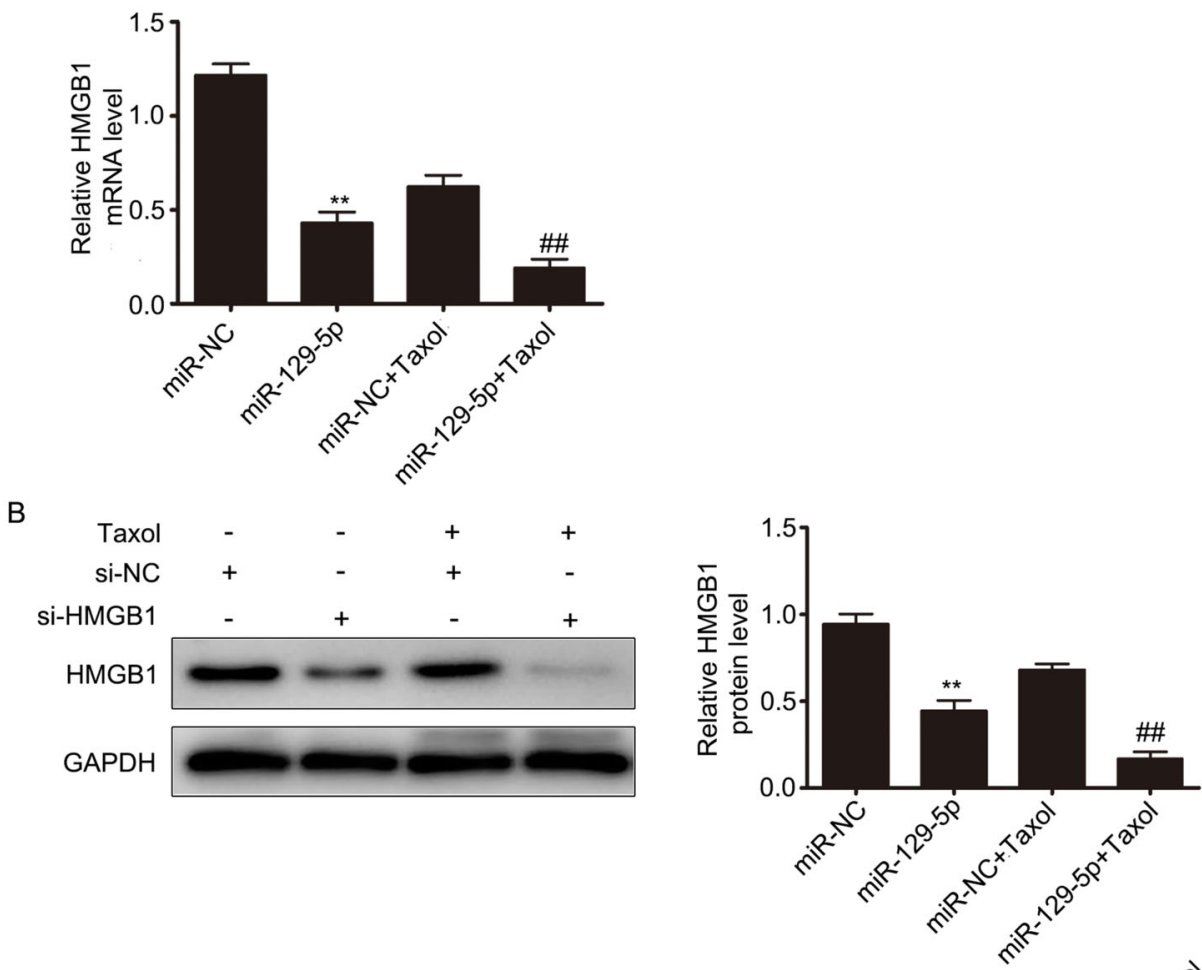

C

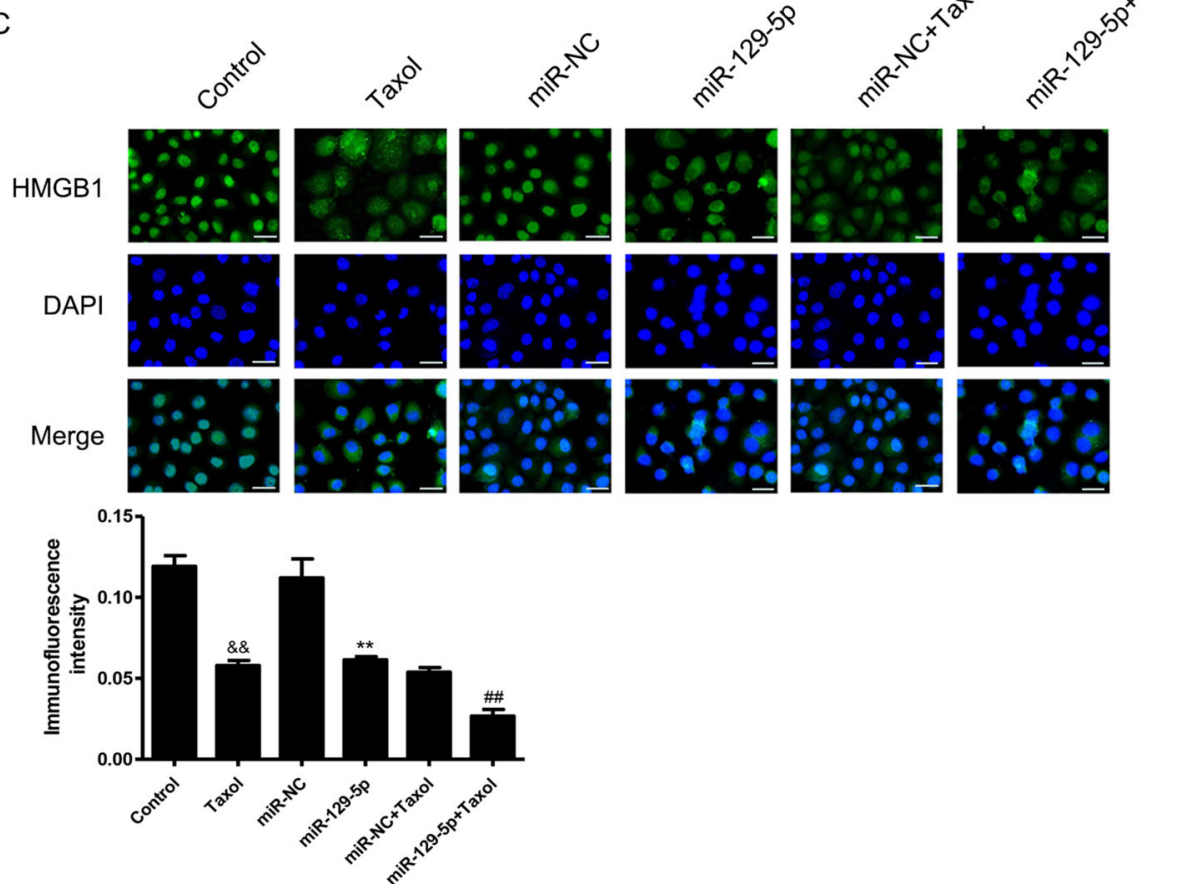

Figure 4. MiR-129-5p was negatively correlated with HMGB1 level. A, B, and C, Cells were transfected with miR-NC or miR-129-5p mimics, and then treated with $31.2 \mathrm{nM}$ Taxol for $24 \mathrm{~h}$. The levels of HMGB1 were determined by qRT-PCR (A), western blotting (B), and immunofluorescence assay (C). Scale bar, $100 \mu \mathrm{m}$. Data are reported as means $\pm \mathrm{SD}$ of three independent experiments. ${ }^{* *} \mathrm{P}<0.01, v s$ miR-NC group; ${ }^{\# \#} \mathrm{P}<0.01$, vs miR-NC + Taxol group; ${ }^{{ }^{\&} \mathrm{P}}<0.01$, vs control group (ANOVA). 
A

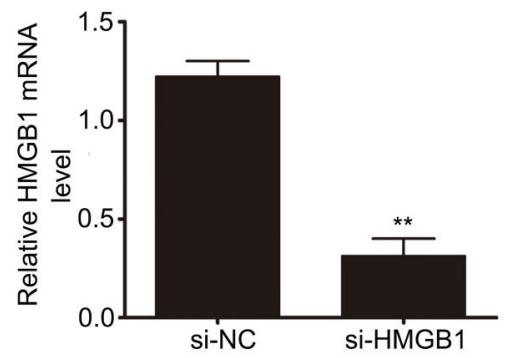

C

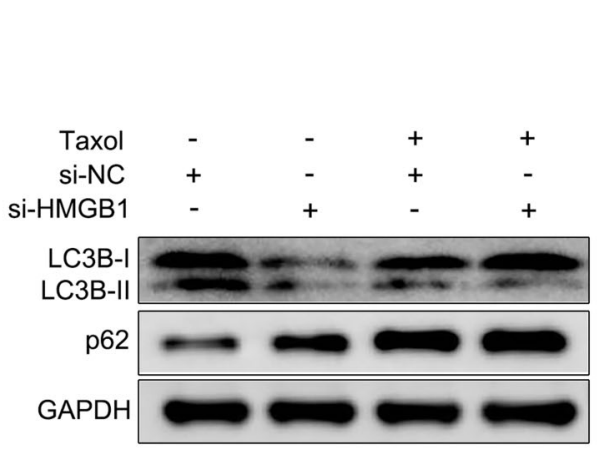

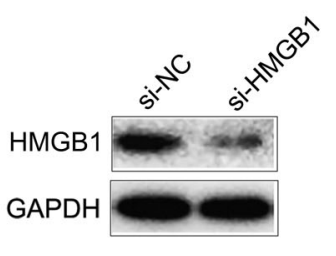

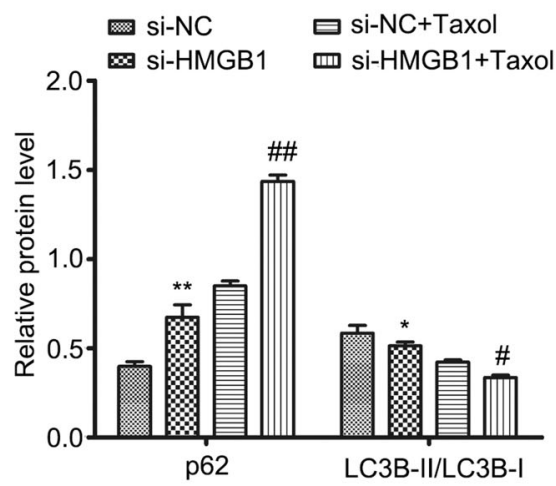

Figure 5. Interference of HMGB1 enhanced chemosensitivity of Taxol by inhibiting autophagy in MCF-7 cells. MCF-7 cells were transfected with si-HMGB1 or si-NC. A, Relative expression of HMGB1 mRNA was detected by qRT-PCR. B, Relative expression of HMGB1 protein was detected by western blot. C, MCF-7 cells were transfected with si-HMGB1 or si-NC and then treated with $31.2 \mathrm{nM}$ Taxol for $24 \mathrm{~h}$. LC3B-I, LC3B-II, and p62 expressions in cells were determined via western blot. Data are reported as means \pm SD of three independent experiments. ${ }^{*} \mathrm{P}<0.05$, ${ }^{*} \mathrm{P}<0.01$, vs si-NC group; ${ }^{\#} \mathrm{P}<0.05,{ }^{\# \#} \mathrm{P}<0.01$, vs si-NC + Taxol group (ANOVA).

present study, we found that miR-129-5p increased the Taxol-induced apoptosis while inhibiting autophagy. Importantly, miR-129-5p increased the sensitivity of MCF-7 cells to Taxol by inhibiting autophagy. Further research demonstrated that miR-129-5p might inhibit autophagy by regulating HMGB1 and we confirmed this result in MCF-7 cells. Thus, we concluded that miR-129-5p increased the sensitivity of Taxol through inhibiting autophagy by regulating HMGB1. Furthermore, miR-129-5p/HMGB1/autophagy might be developed as a novel method for overcoming Taxol resistance in breast cancer.

Autophagy is a double-edged sword. Persistent and excessive autophagy can lead to tumor shrinkage and cell death. However, autophagy can protect cancer cells to maintain their survival in stress conditions $(22,24)$. Recently, many studies have reported that autophagy was a significant factor in the development of drug resistance in breast cancer (25). In this study, we found that Taxol might exert its anti-cancer effect by inhibiting autophagy, implying that the level of autophagy might be associated with the sensitivity of Taxol. To validate this conclusion, we used 3-MA to inhibit autophagy. The results indicated that the inhibition of autophagy could increase the sensitivity of MCF-7 cells to Taxol and the level of apoptosis. These results suggested that autophagy had a pro-survival role in Taxol treatment of breast cancer cells. Meanwhile, autophagy had an anti-apoptosis effect.

To date, an increasing number of miRNAs have been identified to have a close relationship with the sensitivity of Taxol through modulating autophagy or apoptosis $(36,37)$. Yet, the function of miR-129-5p in breast cancer and chemosensitivity is rarely reported. Recently, research demonstrated that miR-129-5p might play multiple and important roles in autophagy processes and drug resistance (38).

Generally, miRNAs play their biological role by targeting gene expression. We found HMGB1 was a target gene of miR-129-5p by searching online analysis tools and databases. Studies also have identified that miR-129-5p directly targets HMGB1 in breast cancer and osteosarcoma $(33,39)$. Thus, it implies that miR129-5p might enhance the sensitivity of Taxol by inhibiting HMGB1.

Recently, researchers reported that HMGB1 contributed to drug sensitivity of cancers by modulating autophagy and apoptosis (40). In our current study, we found HMGB1 was highly expressed in breast cancer by searching databases of Oncomine and the Human Protein Atlas. Many studies have also reported this result in breast cancers. Thus, our study explored the functions of HMGB1 on the sensitivity of 
A
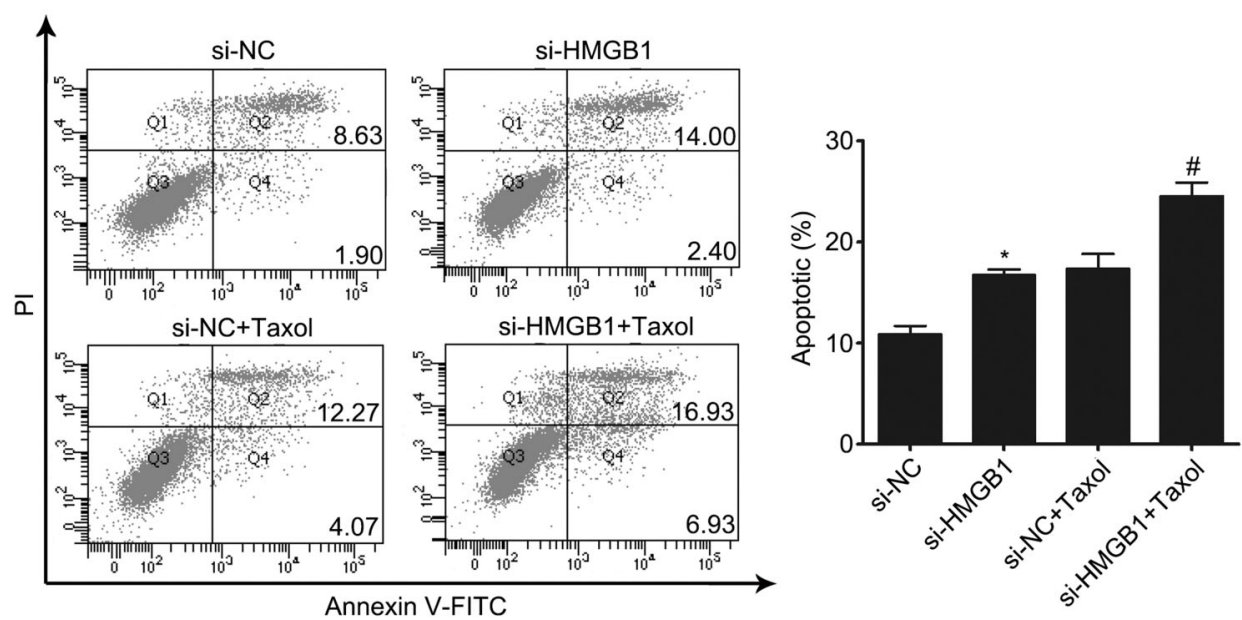

B

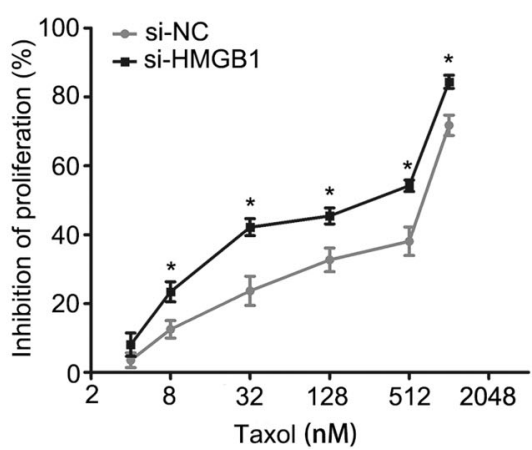

Figure 6. Interference of HMGB1 enhanced chemosensitivity of Taxol by promoting apoptosis in MCF-7 cells. A, Cellular apoptosis was determined by flow cytometry. The total rate of apoptotic cells is represented in a bar diagram from three independent experiments (right panel). B, Cells were transfected with si-NC or si-HMGB1, and then treated with different concentrations of Taxol for $24 \mathrm{~h}$. Cell proliferation was determined by the CCK-8 assay. Data are reported as means $\pm \mathrm{SD}$ of three independent experiments. ${ }^{*} \mathrm{P}<0.05$, vs si-NC group; ${ }^{\#} \mathrm{P}<0.05$, vs si-NC + Taxol group (ANOVA).

Taxol. We found that changing the expression of HMGB1 could decrease the level of Taxol-mediated autophagy. More importantly, our data showed that downregulation of HMGB1 increased the Taxol-induced apoptosis and the sensitivity of MCF-7 cells to Taxol. These results indicated that inhibiting the expression of HMGB1 could improve the sensitivity of MCF-7 cells to Taxol by inhibiting autophagy and promoting apoptosis. However, this experiment used a single cell line, and such observations should be verified in a multi-cell line study design.

In conclusion, our study provides evidence that miR129-5p overexpression increased the chemosensitivity of MCF-7 cells to Taxol through suppressing autophagy and promoting apoptosis by inhibiting HMGB1. This study may

\section{References}

1. Shioi $Y$, Kashiwaba $M$, Inaba $T$, Komatsu $H$, Sugai $T$, Wakabayashi G. Long-term complete remission of metastatic breast cancer, induced by a steroidal aromatase help to better understand the molecular mechanisms of drug resistance in breast cancer, and it may imply that overexpression of miR-129-5p alone or in conjunction with HMGB1 interference, is a promising strategy to combat multidrug resistance in breast cancer.

\section{Supplementary material}

Click here to view [pdf].

\section{Acknowledgments}

This work was supported by the National Natural Science Foundation of China. inhibitor after failure of a non-steroidal aromatase inhibitor. Am J Case Rep 2014; 15: 85-89, doi: 10.12659/AJCR. 890023. 
2. Gianni L, Baselga J, Eiermann W, Porta VG, Semiglazov V, Lluch $A$, et al. Phase III trial evaluating the addition of paclitaxel to doxorubicin followed by cyclophosphamide, methotrexate, and fluorouracil, as adjuvant or primary systemic therapy: European cooperative trial in operable breast cancer. J Clin Oncol 2009; 27: 2474-2481, doi: 10.1200/JCO.2008.19.2567.

3. Erol K, Baltali E, Altundag K, Guler N, Ozisik Y, Onat DA, et al. Neoadjuvant chemotherapy with cyclophosphamide, mitoxantrone, and 5-fluorouracil in locally advanced breast cancer. Onkologie 2005; 28: 81-85, doi: 10.1159/000082951.

4. Gradishar WJ, Anderson BO, Balassanian R, Blair SL, Burstein $\mathrm{HJ}$, Cyr A, et al. Invasive breast cancer version 1.2016, NCCN clinical practice guidelines in oncology. J Natl Compr Canc Netw 2016; 14: 324-354, doi: 10.6004/ jnccn.2016.0037.

5. Schiff PB, Horwitz SB. Taxol stabilizes microtubules in mouse fibroblast cells. Proc Natl Acad Sci USA 1980; 77: 1561-1565, doi: 10.1073/pnas.77.3.1561.

6. Schiff PB, Fant J, Horwitz SB. Promotion of microtubule assembly in vitro by Taxol. Nature 1979; 277: 665-667, doi: $10.1038 / 277665 \mathrm{a} 0$.

7. Rowinsky EK. The development and clinical utility of the taxane class of antimicrotubule chemotherapy agents. Annu Rev Med 1997; 48: 353-374, doi: 10.1146/annurev.med. 48.1.353.

8. Wang H, Vo T, Hajar A, Li S, Chen X, Parissenti AM, et al. Multiple mechanisms underlying acquired resistance to taxanes in selected docetaxel-resistant MCF-7 breast cancer cells. BMC Cancer 2014; 14: 37, doi: 10.1186/ 1471-2407-14-37.

9. Zelnak A. Overcoming taxane and anthracycline resistance. Breast J 2010; 16: 309-312, doi: 10.1111/j.1524-4741.2010. 00911.x.

10. Friedman RC, Farh KK, Burge CB, Bartel DP. Most mammalian mRNAs are conserved targets of microRNAs. Genome Res 2008; 19: 92-105, doi: 10.1101/gr.082701.108.

11. Chen GS, Zhou N, Li JQ, Li T, Zhang ZQ, Si ZZ. Restoration of miR-20a expression suppresses cell proliferation, migration, and invasion in HepG2 cells. Onco Targets Ther 2016; 9: 3067-3076, doi: 10.2147/OTT.S96861.

12. Bhattacharya A, Schmitz U, Raatz Y, Schönherr M, Kottek T, Schauer M, et al. MiR-638 promotes melanoma metastasis and protects melanoma cells from apoptosis and autophagy. Oncotarget 2015; 6: 2966-2980, doi: 10.18632/oncotarget. 3070.

13. Li S, Qiang Q, Shan H, Shi M, Gan G, Ma F, et al. MiR-20a and miR-20b negatively regulate autophagy by targeting RB1CC1/FIP200 in breast cancer cells. Life Sci 2016; 147: 143-152, doi: 10.1016/j.Ifs.2016.01.044.

14. Chen C, Hu Y, Li L. NRP1 is targeted by miR-130a and miR$130 \mathrm{~b}$, and is associated with multidrug resistance in epithelial ovarian cancer based on integrated gene network analysis. Mol Med Report 2016; 13: 188-196, doi: 10.3892/ mmr.2015.4556.

15. Chen QY, Jiao DM, Wang J, Hu H, Tang $X$, Chen J, et al. miR-206 regulates cisplatin resistance and EMT in human lung adenocarcinoma cells partly by targeting MET. Oncotarget 2016; 7: 24510-24526, doi: 10.18632/oncotarget.8229.

16. Wang $\mathrm{Y}$, Lieberman R, Pan J, Zhang Q, Du M, Zhang P, et al. MiR-375 induces docetaxel resistance in prostate cancer by targeting SEC23A and YAP1. Mol Cancer 2016; 15: 70, doi: 10.1186/s12943-016-0556-9.

17. Bo R, Park W. Suberoylanilide hydroxamic acid induces thioredoxin1-mediated apoptosis in lung cancer cells via upregulation of miR-129-5p. Mol Carcinog 2017; 56: 25662577, doi: $10.1002 / \mathrm{mc} .22701$

18. $\mathrm{Xu} \mathrm{H}, \mathrm{Hu} Y$, Qu W. Potential mechanisms of microRNA$129-5 p$ in inhibiting cell processes including viability, proliferation, migration and invasiveness of glioblastoma cells U87 through targeting FNDC3B. Biomed Pharmacother 2017; 87: 405-411, doi: 10.1016/j.biopha.2016.12.100.

19. Wang Q, Yu J. MiR-129-5p suppresses gastric cancer cell invasion and proliferation by inhibiting COL1A1. Biochem Cell Biol 2018; 96: 19-25, doi: 10.1139/bcb-2016-0254.

20. Yu Y, Zhao Y, Sun XH, Ge J, Zhang B, Wang X, et al. Downregulation of miR-129-5p via the Twist1-Snail feedback loop stimulates the epithelial-mesenchymal transition and is associated with poor prognosis in breast cancer. Oncotarget 2015; 6: 34423-34436, doi: 10.18632/oncotarget.5406.

21. Geng Z, Xu F, Zhang Y. Mir-129-5p-mediated beclin-1 suppression inhibits endothelial cell autophagy in atherosclerosis. Am J Transl Res 2016; 8: 1886-1894.

22. Liu K, Huang J, Xie M, Yu Y, Zhu S, Kang R, et al. MiR34A regulates autophagy and apoptosis by targeting HMGB1 in the retinoblastoma cell. Autophagy 2014; 10: 442-452, doi: 10.4161/auto. 27418 .

23. Luan QX, Zhang BG, Li XJ, Guo MY. Mir-129-5p is downregulated in breast cancer cells partly due to promoter h3k27m3 modification and regulates epithelial-mesenchymal transition and multi-drug resistance. Eur Rev Med Pharmacol Sci 2016; 20: 4257-4265.

24. Thorburn A. Autophagy and its effects: making sense of double-edged swords. PLoS Biol 2014; 12: e1001967, doi: 10.1371/journal.pbio.1001967.

25. Kubisch J, Türei D, Földvári-Nagy L, Dunai ZA, Zsákai L, Varga $\mathrm{M}$, et al. Complex regulation of autophagy in cancerintegrated approaches to discover the networks that hold a double-edged sword. Semin Cancer Biol 2013; 23: 252261, doi: 10.1016/j.semcancer.2013.06.009.

26. Liu Z, He K, Ma Q, Yu Q, Liu C, Ndege I, et al. Autophagy inhibitor facilitates gefitinib sensitivity in vitro and in vivo by activating mitochondrial apoptosis in triple negative breast cancer. PLoS One 2017; 12: e0177694, doi: 10.1371/ journal.pone. 0177694 .

27. Circu M, Cardelli J, Barr MP, O’Byrne K, Mills G, El-Osta H. Modulating lysosomal function through lysosome membrane permeabilization or autophagy suppression restores sensitivity to cisplatin in refractory non-small-cell lung cancer cells. PLoS One 2017; 12: e0184922, doi: 10.1371/journal. pone.0184922.

28. Melvin VS, Harrell C, Adelman JS, Kraus WL, Churchill M, Edwards DP. The role of the C-terminal extension (CTE) of the estrogen receptor alpha and beta DNA binding domain in DNA binding and interaction with HMGB. $J$ Biol Chem 2004; 279: 14763-14771, doi: 10.1074/jbc.M313335200.

29. Yung F, Gu L, Guo S, Wang C, Li GM. Evidence for involvement of HMGB1 protein in human DNA mismatch repair. J Biol Chem 2004; 279: 20935-20940, doi: 10.1074/ jbc.M401931200.

30. Guo X, He D, Zhang E, Chen J, Chen Q, Li Y, et al. HMGB1 knockdown increases $\mathrm{MM}$ cell vulnerability by regulating 
autophagy and DNA damage repair. J Exp Clin Cancer Res 2018; 37: 205, doi: 10.1186/s13046-018-0883-3.

31. Ai H, Zhou W, Wang Z, Qiong G, Chen Z, Deng S. MicroRNAs-107 inhibited autophagy, proliferation, and migration of breast cancer cells by targeting HMGB1. J Cell Biochem 2018, doi: 10.1002/jcb.28157.

32. Liu Z, Du R, Long J, Guo K, Ge C, Bi S, et al. MicroRNA218 promotes gemcitabine sensitivity in human pancreatic cancer cells by regulating HMGB1 expression. Chin $J$ Cancer Res 2015; 27: 267-278, doi: 10.3978/j.issn.10009604.2015.04.06.

33. Luo J, Chen J, He L. MiR-129-5p Attenuates irradiationinduced autophagy and decreases radioresistance of breast cancer cells by targeting HMGB1. Med Sci Monit 2015; 21: 4122-4129, doi: 10.12659/MSM.896661.

34. Schmeisser H, Bekisz J, Zoon KC. New function of type I IFN: induction of autophagy. J Interferon Cytokine Res 2014; 34: 71-78, doi: 10.1089/jir.2013.0128.

35. Korkmaz G, le Sage C, Tekirdag KA, Agami R, Gozuacik D. MiR-376b controls starvation and mTOR inhibitionrelated autophagy by targeting ATG4C and BECN1. Autophagy 2012; 8: 165-176, doi: 10.4161/auto.8.2. 18351.
36. Chatterjee A, Chattopadhyay D, Chakrabarti G. MiR-16 targets $\mathrm{Bcl}-2$ in paclitaxel-resistant lung cancer cells and overexpression of miR-16 along with miR-17 causes unprecedented sensitivity by simultaneously modulating autophagy and apoptosis. Cell Signal 2015; 27: 189-203, doi: 10.1016/j.cellsig.2014.11.023.

37. Zhao ZL, Cai Y, Wang YY, Xia CL, Li CX, Chen SL, et al. Effects of miRNA-21 on paclitaxel-resistance in human breast cancer cells. Zhejiang Da Xue Xue Bao Yi Xue Ban 2015; 44: 400-409.

38. Ma Z, Cai H, Zhang Y, Chang L, Cui Y. MiR-129-5p inhibits non-small cell lung cancer cell stemness and chemoresistance through targeting DLK1. Biochem Biophys Res Commun 2017; 490: 309-316, doi: 10.1016/j.bbrc.2017.06.041.

39. Liu K, Huang J, Ni J, Song D, Ding M, Wang J, et al. Malati1 promotes osteosarcoma development by regulation of HMGB1 via miR-142-3p and miR-129-5p. Cell Cycle 2017; 16: 578-587, doi: 10.1080/15384101.2017.1288324.

40. Liu W, Zhang Z, Zhang Y, Chen X, Guo S, Lei Y, et al. HMGB1-mediated autophagy modulates sensitivity of colorectal cancer cells to oxaliplatin via MEK/ERK signaling pathway. Cancer Biol Ther 2015; 16: 511-517, doi: 10.1080/ 15384047.2015.1017691. 